\title{
TOPOLOGIES AND PROJECTIONS ON RIESZ SPACES
}

\author{
BY \\ HUGH GORDON(1)
}

Introduction. Among the various questions about topologies on Riesz spaces, there is a particularly important one which arises out of Daniell's work on integration. The present paper will be centered around this question, but will not be confined to it. Daniell [5] considers a space $V$ whose elements are real-valued functions on an abstract set $E$ and which is closed under the natural linear and lattice operations. He starts with a positive linear functional $F$ on $V$ (that is, $F f \geqq 0$ whenever $f(x) \geqq 0$ for all $x \in E$ ) endowed with the property that $f_{n}(x) \uparrow f(x)$ pointwise implies $F f_{n} \rightarrow F f$. Daniell extends $F$ from $V$ to a larger class of functions in such a way that the extended $F$ satisfies the theorem of Lebesgue: Under suitable conditions of boundedness, if $f_{n} \rightarrow f$ pointwise and the $F f_{n}$ are defined, $F f$ is defined and $F f_{n} \rightarrow F f$. It follows that the extended $F$ is the integral corresponding to a measure on $E$.

In accordance with the work of Daniell, we call a positive linear functional $F$ on $V$ an integral if it has the property: $f_{n} \uparrow f$ pointwise implies $F f_{n} \rightarrow F f$. By an integral we mean any functional which is the difference of two positive integrals.

The relationship of the set of integrals to the set of all functionals was considered by Riesz [10, p. 206]. He considered the class of positive functionals which exceed no positive integral other than zero. He showed that any positive functional may be expressed as the sum of a functional of this class and an integral.

The result of Riesz employs what is essentially the notion of a direct sum. Of course we may replace that notion by the equivalent one of a projection. The problem is considered from this point of view by Gordon and Lorch [7]. Suppose the functions in $V$ are bounded. We define a norm on $V$ by $\|f\|=1$.u.b. $x \in E|f(x)|$. Suppose $V$ is a Banach space, i.e. suppose $V$ is complete in its norm. Let $V^{*}$ be the space of all continuous linear functionals on $V$. Then these authors prove there is a unique projection $P$ (i.e. an idempotent linear mapping) of $V^{*}$ into itself with the properties that:

(a) The range of $P$ is precisely the set of integrals.

(b) $0 \leqq P F f \leqq F f$ whenever $F \geqq 0$ and $f \geqq 0$.

Presented to the Society, April 5, 1957 under the title Compatible topologies on Riesz spaces. Preliminary report; received by the editors June 2, 1958 and, in revised form, March 28, 1959.

(1) This paper consists of a portion of the author's doctoral dissertation, which was prepared at Columbia University under the direction of Professor E. R. Lorch. Part of this work was done under National Science Foundation grant NSF G 1981. 
They give an explicit formula for $P F f$ in terms of $F$ and $f$.

This question has also been considered by $H$. Bauer [1], who retains Riesz's point of view. Bauer replaces the space of functions by a lattice and the linear functionals by valuations. He uses arbitrary monotone directed sequences, in the manner indicated below, instead of monotone pointwise convergent sequences. In these circumstances he establishes the existence of direct sum decompositions. In another paper relating to other matters, he gives a formula which may be interpreted as the formula for $P F f$ referred to above [2, p. 455].

We may look upon the situation described above as being roughly as follows: We have a vector space of functions $V$ with a topology. This gives rise to the space $V^{*}$ of all linear functionals which are continuous over $V$. A submanifold of $V^{*}$ (above the integrals) is singled out by the requirement that the functionals of this submanifold be continuous over $V$ in another topology. In the case of integration, the topology is not precisely defined; it is hinted at by the condition of continuity: $f_{n} \uparrow f \Rightarrow F f_{n} \rightarrow F f$. The results above then state that we have in this special situation a projection of the manifold of all functionals onto the manifold of those continuous in the second topology. The question to which the Daniell program gives rise (and to which we alluded in the first paragraph of this paper) is to provide a proper setting for the generalization of these results and to give necessary and sufficient conditions for the existence and uniqueness of the projection.

The proper "space setting" seems clearly to be that $V$ should be a topological Riesz space. The notion of a compatible topology on a Riesz space is considered below. (Such topologies were also used by Roberts [11] and by Namioka [9]. Their main results are not relevant to our undertaking and we shall not presuppose any of their work.)

The problem may be precisely stated as follows: Let a topological Riesz space $V$ be given. Let $V^{*}$ be the set of its continuous linear functionals. Suppose there is given on $V$ a second topology compatible with its Riesz space structure. Let $M$ be the subset of $V^{*}$ consisting of those functionals which are continuous in the second topology, as well as the original one. When does there exist a projection $P$ defined on $V^{*}$ such that:

(a) The range of $P$ is $M$.

(b) $0 \leqq P F f \leqq F f$ whenever $F \geqq 0$ and $f \geqq 0$.

We give a necessary and sufficient condition for the existence of such a projection (see in particular Theorem 3.11).

As noted above, the integrals are not defined as the continuous functionals for a genuine topology, but rather as the functionals satisfying a certain continuity condition involving sequences. More precisely, a positive linear functional $F$ is an integral if $F f_{n} \rightarrow 0$ whenever $\left(f_{1}, f_{2}, \cdots\right)$ belongs to a certain collection of sequences $\mathcal{S}$. $\mathcal{S}$ consists of those sequences such that $f_{n} \downarrow 0$ pointwise. Now let $V$ be an arbitrary Riesz space and let $V^{*}$ be the set of 
linear functionals continuous in some compatible topology on $V$. Let $\delta$ be an arbitrary collection of directed sequences of elements in $V$; let $M$ be the manifold of those linear functionals in $V^{*}$ which are $s$-continuous, that is, such that $\left\{f_{\imath}\right\}_{\iota \in I} \in S \Rightarrow F f_{\imath} \rightarrow 0$. As before, we seek to determine whether there exists a projection of $V^{*}$ onto $M$. This problem is given a solution by Theorems 4.6 and 4.7. We shall show that there is a projection if each sequence in $S$ is bounded; thus any collection of bounded sequences leads to a projection. Conversely, if $V, V^{*}$ and a projection on $V^{*}$ are given, we can find a collection $S$ of bounded sequences such that the manifold $M$ of $\mathcal{S}$-continuous functionals is precisely the range of the projection; thus any projection leads to a collection of bounded sequences.

1. Compatible topologies on Riesz spaces $\left({ }^{2}\right)$. In this section we consider the basic properties of Riesz spaces with compatible topologies. Some of the results will be needed in the following sections and all of them will be relevant there. Most of the material relates to questions involving duality and is of interest in itself. Theorems 1.14 and 1.18 are especially important to the present development.

Let $V$ be a Riesz space. (I.e. $V$ is a lattice ordered real vector space.) We shall say that a topology is compatible with the Riesz space structure of $V$ provided the following axioms are satisfied:

Aхгом 1. The topology is compatible with the vector space structure of $V$.

Axıом 2. The mapping $f \rightarrow f^{+}$of $V$ into itself is continuous at 0 .

Axiom 3. For each neighborhood $U$ of 0 there is a neighborhood $U^{\prime}$ of 0 with the following property:

$$
\text { If } f \in U^{\prime}, g \in V \text { and } 0 \leqq g \leqq f, \text { then } g \in U \text {. }
$$

A Riesz space endowed with a compatible topology will be called a topological Riesz space: If $V$ is a topological Riesz space, it has the following property:

1.1. TheOREM $\left({ }^{3}\right)$. The mappings $(f, g) \rightarrow f \bigvee g$ and $(f, g) \rightarrow f \wedge g$ of $V \times V \rightarrow V$ are continuous.

Proof. We first show that the mapping $f \rightarrow|f|$ is continuous at 0 . Clearly $f \rightarrow f^{-}=(-f)^{+}$is continuous at 0 ; hence $f \rightarrow|f|=f^{+}+f^{-}$is continuous at 0 .

We next prove that the mapping $f \rightarrow f^{+}$is continuous everywhere. Let $g \in V$. Let $U$ be a neighborhood of $g^{+}$. Then $W_{1}=U-g^{+}$is a neighborhood of 0 . Let $W_{2}$ be a neighborhood of 0 such that $W_{2}-W_{2} \subset W_{1}$. Let $W_{3}$ be a neigh-

(2) The material of this section was described in [6].

(3) After the abstract of [6] was submitted, Namioka [9, pp. 18 and 40], showed that the system of axioms above is equivalent to the following system:

Axiom 1': Same as Axiom 1.

Axiom 2': Same as Theorem 1.1.

Axiom $3^{\prime}$ : Each neighborhood of 0 contains a neighborhood $U$ of 0 such that: If $f \in U$, $g \in V$ and $0 \leqq g \leqq f$, then $g \in U$. 
borhood of 0 such that $0 \leqq h \leqq k \in W_{3}$ implies $h \in W_{2}$. By the preceding paragraph, there is a neighborhood $W_{4}$ of 0 such that $\left|W_{4}\right| \subset W_{3}$. Let $U^{\prime}=W_{4}+g$. We note $U^{\prime}$ is a neighborhood of $g$. Let $f \in U^{\prime}$. Then $f-g \in W_{4}$; thus $|f-g|$ $\in W_{3}$. We recall from $\left[3\right.$, p. 220], that $\left|f^{+}-g^{+}\right| \leqq|f-g|$. Thus, since $0 \leqq\left(f^{+}-g^{+}\right)^{+} \leqq\left|f^{+}-g^{+}\right| \leqq|f-g| \in W_{3},\left(f^{+}-g^{+}\right)^{+} \in W_{2}$. Similarly $\left(f^{+}-g^{+}\right)^{-}$ $\in W_{2}$. Thus $f^{+}-g^{+}=\left(f^{+}-g^{+}\right)^{+-}\left(f^{+}-g^{+}\right)^{-} \in W_{1}$. Therefore $f^{+} \in W_{1}+g^{+}=U$. Thus, for every $f \in U^{\prime}, f^{+} \in U$; hence $f \rightarrow f^{+}$is continuous at $g$ for each $g \in V$.

To prove the theorem, it is now enough to note $f \vee g=f+(g-f)^{+}$and $f \wedge g=f-(f-g)^{+}$.

Let $V$ be a Riesz space with a topology given by a norm. Suppose $\|f\| \leqq\|g\|$ whenever $0 \leqq f \leqq g$. Suppose also $\left\|f^{+}\right\| \leqq\|f\|$ for all $f$. Then the topology is compatible with the Riesz space structure. Similarly, if the topology is given by semi-norms each having the above properties, the topology is compatible.

1.2. Theorem. Every Hausdorff topological Riesz space is an ordered topological vector space.

Proof. The set of positive elements in $V$ is closed because it is the inverse image of 0 under the continuous mapping $f \rightarrow(-f)^{+}$.

In view of the above theorem, the following theorem applies to Hausdorff topological Riesz spaces:

1.3. TheOREM. Let $V$ be an ordered vector space and $\left\{f_{\imath}\right\}_{\iota \in I}$ be a directed sequence of elements of $V$. Suppose $f_{\iota} \leqq f_{\nu}$ whenever $\iota \leqq \nu$. Let $J_{1}, J_{2}$ be topologies on $V$ compatible with the ordered vector space structure of $V$. Then:

(a) If $\left\{f_{\iota}\right\}_{\iota \in I}$ is $J_{1}$-convergent to $f, f_{\iota} \leqq f$ for all $\iota \in I$.

(b) If $\left\{f_{\imath}\right\}_{\iota \in I}$ is $J_{1}$-convergent to $f$ and $f_{\iota} \leqq g$ for all $\iota \in I, f \leqq g$.

(c) If $\left\{f_{\iota}\right\}_{\iota \in I}$ is both $J_{1}$-convergent to $g_{1}$ and $J_{2}$-convergent to $g_{2}, g_{1}=g_{2}$.

Proof. (a) Suppose $f_{\mathfrak{c}}$ is not $\leqq f$. Let $U$ be a neighborhood of $f$. The set $A$ of all $h \geqq f_{\imath}$ is closed; $f \notin A$. $U-A$ is thus a neighborhood of $f$. Hence $f_{\nu} \in U-A$ for all $\nu$ sufficiently large. But $f_{\nu} \in A$ for all $\nu \geqq \iota$; thus $f_{\nu} \in U-A$ for large $\nu$.

(b) The set $A$ of all $h \leqq g$ is closed. $f_{\iota} \in A$ for all $\iota$; hence $f \in A$.

(c) By (a) we have $f_{\iota} \leqq g_{1}$ for all $\iota$. Hence, applying (b) to $J_{2}$ instead of $J_{1}$, we have $g_{2} \leqq g_{1}$. Similarly $g_{1} \leqq g_{2}$; thus $g_{1}=g_{2}$.

1.4. Theorem. Let $V_{1}, V_{2}$ be topological Riesz spaces. Let $\phi_{1}, \phi_{2}$ be mappings of $V_{1}$ into $V_{2}$. Suppose that $\phi_{2}$ is continuous at 0 and that $\phi_{1}$ is linear. Suppose also that for each positive $f \in V_{1}, 0 \leqq \phi_{1}(f) \leqq \phi_{2}(f)$, and that $\phi_{2}(0)=0$. Then $\phi_{1}$ is continuous.

Proof. Let $U$ be any neighborhood of 0 in $V_{2}$. Let $U_{1}, U_{2}$ be neighborhoods of 0 in $V_{2}$ such that $U_{1}-U_{2} \subset U$. Let $U_{i}^{\prime}(i=1,2)$ be such that if $0^{\prime} \leqq h \leqq k \in U_{i}^{\prime}$ then $h \in U_{i}$. Let $W_{1}$ be the inverse image of $U_{1}^{\prime}$ under the mapping $f \rightarrow \phi_{2}\left(f^{+}\right)$; let $W_{2}$ be the inverse image of $U_{2}^{\prime}$ under the mapping $f \rightarrow \phi_{2}\left(f^{-}\right)$. Let $W=W_{1} \cap W_{2}$. We note that $W$ is a neighborhood of 0 in $V_{1}$ since $W_{1}$ and $W_{2}$ 
are. Hence, to prove the theorem, it is enough to show $\phi_{1}(W) \subset U$. Let $f \in W$. We have $0 \leqq \phi_{1}\left(f^{+}\right) \leqq \phi_{2}\left(f^{+}\right) \in U_{1}^{\prime}$ since $f \in W \subset W_{1}$. Thus $\phi_{1}\left(f^{+}\right) \in U_{1}$. Similarly $\phi_{2}\left(f^{-}\right) \in U_{2}$. Therefore $\phi_{1}(f)=\phi_{1}\left(f^{+}-f^{-}\right)=\phi_{1}\left(f^{+}\right)-\phi_{1}\left(f^{-}\right) \in U_{1}-U_{2}$ $\subset U$.

1.5. Corollary. Let $V$ be a topological Riesz space and $F_{1}, F_{2}$ be linear functionals on $V$. If $F_{2}$ is continuous and $0 \leqq F_{1} \leqq F_{2}$, then $F_{1}$ is continuous.

1.6. Corollary. Let $V$ be a topological Riesz space and $P$ be a linear mapping of $V$ into $V$. If $P$ and $I-P$ ( $I$ is the identity mapping of $V$ onto $V$ ) are positive, $P$ is continuous.

Proof. $I$ is continuous and $0 \leqq P \leqq I$.

We now proceed to study continuous real linear functionals on a topological Riesz space $V$.

1.7. Lemma. If $A \subset V$ is such that $0 \leqq g \leqq f$ for all $g \in A$ and some $f \in V$, then $A$ is bounded in the topological vector space sense.

Proof. Let $U$ be any neighborhood of 0 . Let $U^{\prime}$ be such that if $0 \leqq h \leqq k$ and $k \in U^{\prime}$ then $h \in U$. For $\alpha>0$ sufficiently small, $\alpha f \in U^{\prime}$. For every $g \in A$, $0 \leqq \alpha g \leqq \alpha f \in U^{\prime}$, therefore $\alpha A \subset U$. Thus $A$ is bounded.

We recall that a linear functional $F$ is called relatively bounded if, for each positive $f \in V$, the set $\{|F g||| g \mid \leqq f, g \in V\}$ is bounded; it has been shown in [3, p. 245], that a functional is relatively bounded if and only if it is the difference of two positive functionals.

1.8. THEOREM. Every continuous linear functional on $V$ is relatively bounded.

Proof. Let $F$ be a continuous linear functional on $V$. Let $f \in V$ be positive. Let $A$ be the set of $g \in V$ such that $0 \leqq g \leqq f$. By the lemma, $A$ is topologically bounded. Since $F$ is continuous, there is a neighborhood $U$ of 0 such that $|F h|<1$ for all $h \in U$. Since $A$ is bounded, $\alpha A \subset U$ for some $\alpha>0$. Thus if $g \in A, \quad|F g|=(1 / \alpha)|F(\alpha g)|<1 / \alpha$ since $\alpha g \in U$. Therefore $F$ is relatively bounded.

Since every continuous linear functional $F$ is relatively bounded, we know that for each $F$ there are linear functionals $F^{+}, F^{-}$such that $F=F^{+}$ $-F^{-}$. We now prove that $F^{+}$and $F^{-}$are continuous and thus establish that the set of continuous linear functionals is a Riesz space.

1.9. Lemma. If $F$ is a continuous linear functional on $V$, then $F^{+}, F^{-}$are continuous.

Proof. We recall from [3, p. 245], that $F^{+} f=1$.u.b. $0 \leq o \leq f ~ F g$ for $f \geqq 0$. It is enough to prove $F^{+}$is continuous at 0 . Let $\epsilon>0$ be given. Let $U$ be a neighborhood of 0 such that $|F f|<\epsilon / 2$ for all $f \in U$. Let $U^{\prime}$ be a neighborhood of 0 such that if $0 \leqq h \leqq k \in U^{\prime}, h \in U$. Let $W_{1}, W_{2}$ be neighborhoods of 0 such 
that if $f \in W_{1}, f^{+} \in U^{\prime}$ and if $f \in W_{2}, f^{-} \in U^{\prime}$. Let $W=W_{1} \cap W_{2}$. Consider any $f \in W$. We note $f^{+} \in U^{\prime}$ and $f^{-} \in U^{\prime}$. Thus if $0 \leqq g \leqq f^{+}, g \in U$. Also if $0 \leqq g \leqq f^{-}$, $g \in U$. We have for all $f \in W:\left|F^{+} f\right|=\left|F^{+} f^{+}-F^{+} f^{-}\right|=\mid$1.u.b.0sgs $f^{+} F g$

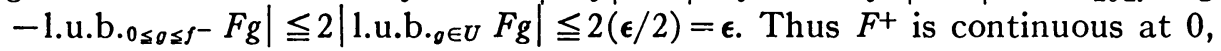
hence everywhere. Since $F^{\llcorner}=F^{+}-F, F^{-}$is continuous.

Recalling that a Riesz space is called completely reticulated if every set bounded from above has a least upper bound, we prove the following theorem:

1.10. Theorem. The set $V^{*}$ of continuous linear functionals on a topological Riesz space is a completely reticulated Riesz space.

Proof. We already know that $V^{*}$ is a Riesz space. Let $A \subset V^{*}$ be majorized by $F \in V^{*}$; i.e. $H \leqq F$ for each $H \in A$. Suppose $A \neq \varnothing$. Pick any fixed $G \in A$ and let $B=A+G^{-}$. Then $G^{+}=G+G^{-} \in A+G^{-}=B$. Also $H \leqq F+G^{-}$for each $H \in B$. Since the set $V_{R}^{*}$ of all relatively bounded linear functionals on $V$ is completely reticulated (see [10, p. 179]), there is a least upper bound $K \in V_{R}^{*}$ for $B$ with respect to $V_{R}^{*}$. We note that $K \leqq F+G^{-}$since $F+G^{-}$is an upper bound for $B$. Also $0 \leqq G^{+} \leqq K$ since $G^{+} \in B$. Thus $0 \leqq K \leqq F+G^{-}$and $F+G^{-}$ is continuous; hence $K$ is continuous by Corollary 1.5, i.e. $K \in V^{*}$. Therefore $K$ is a least upper bound for $B$ with respect to $V^{*}$. It follows that $K-G^{-} \in V^{*}$ is a least upper bound for $A$. Thus $V^{*}$ is completely reticulated.

Let $V$ be a Riesz space. Let $M$ be a set of positive linear functionals on $V$. Then for each $F \in M$ the mapping $f \rightarrow F|f|$ of $V$ into the real numbers is a semi-norm since:

$$
\begin{aligned}
F|f+g| & \leqq F(|f|+|g|)=F|f|+F|g| \quad(\text { since } F \geqq 0), \\
F|\alpha f| & =F(|\alpha||f|)=|\alpha| F|f| .
\end{aligned}
$$

Let $\Gamma$ be the set of all such semi-norms. Then $\Gamma$ defines on $V$ a topology in which $V$ is a locally convex topological vector space. We shall call this topology the "Riesz space weak topology induced on $V$ by $M$ ".

1.11. Lemma. The Riesz space weak topology induced on $V$ by $M$ is compatible with the Riesz space structure of $V$.

Proof. Axiom 1 is satisfied because the topology is defined by seminorms. We verify Axioms 2 and 3 simultaneously. Let $U$ be a neighborhood of 0 . Then there is a $U^{\prime} \subset U$, real numbers $\alpha_{1}, \cdots, \alpha_{n}>0$, and functionals $F_{1}, \cdots, F_{n} \in M$ such that $f \in U^{\prime}$ if and only if $F_{i}|f| \leqq \alpha_{i}$ for all $i$. If $0 \leqq f$ $\leqq g \in U^{\prime}$, then $F_{i}|f|=F_{i} f \leqq F_{i} g=F_{i}|g| \leqq \alpha_{i}$ for all $i$; i.e. $f \in U^{\prime} \subset U$. Axiom 3 is thus verified. Also, the mapping $\phi: f \rightarrow f^{+}$is such that $\phi\left(U^{\prime}\right) \subset U^{\prime}$ since $F_{i}|\phi(f)|=F_{i} f^{+} \leqq F_{i}|f|$ for all $i$; thus we see that $\phi$ is continuous at 0 , which is Axiom 2.

1.12. THEOREM. The Riesz space weak topology induced on $V$ by $M$ is the coarsest topology in which $V$ is a topological Riesz space and the functionals in $M$ are continuous. 
Proof. We first show that the functionals of $M$ are continuous in this topology. For each $F \in M$, the mapping $\phi: f \rightarrow F|f|$ is clearly continuous at 0 , $\phi(0)=0$ and $0 \leqq F f=\phi(f)$ when $f$ is positive. Thus by Theorem 1.4, $F$ is continuous.

Next let $J$ be any topology compatible with the Riesz space structure of $V$ and such that the functionals in $M$ are J-continuous. Then, for each $F \in M$, the mapping $f \rightarrow F|f|$ is J-continuous. Thus $J$ is clearly finer than the Riesz space weak topology induced on $V$ by $M$.

(We now investigate by means of two examples whether it is true that the Riesz space weak topology induced on $V$ by $M$ is the weak topology induced on $V$ by $M$ (i.e. the coarsest topology in which $V$ is a topological vector space and the functionals in $M$ are continuous). In one example the statement will be true; in the other false. In each case, it will later be clear, by Theorem 1.14, that $M$ is the set of all positive continuous functionals on $V$. In each case, the topology is Hausdorff. In both examples, $V$ is the set of all continuous functions on the closed interval $[0,1]$, ordered in the usual way.

First, for each $x \in[0,1]$, let $F_{x}$ be defined by $F_{x} f=f(x)$. Let $F \in M$ whenever $F$ is positive and there are $x_{1}, \cdots, x_{n} \in[0,1]$ such that $F \leqq F_{x_{1}}+\cdots$ $+F_{x_{n}}$. We note that $\left|F_{x} f\right|=|f(x)|=F_{x}|f|$ for each $f \in V$ and $x \in[0,1]$. Hence if $F \leqq F_{x_{1}}+\cdots+F_{x_{n}},\{f|F| f \mid<\alpha\} \supset \cap_{i=1}^{n}\left\{f|| F_{x_{i}} f \mid<\alpha / n\right\}$. It follows that, in this case, the two topologies mentioned above coincide.

Second, for each pair $y, z \in[0,1]$ with $y<z$, let $F_{y z}$ be defined by $F_{y z} f$ $=\int_{y}^{z} f(x) d x$. Let $F \in M$ whenever $F$ is positive and there are $y_{1}, \cdots, y_{n}$, $z_{1}, \cdots, z_{n} \in[0,1]$ with $y_{i}<z_{i}$ such that $F \leqq F_{y_{1} z_{1}}+\cdots+F_{y_{n} z_{n}}$. Let $f_{m}(x)$ $=\sin (2 \pi m x), m=1,2, \cdots$. Then $F_{01}\left|f_{m}\right|=\int_{0}^{1}|\sin (2 \pi m x)| d x=2 / \pi$ for all $m$. Thus $f_{m} \rightarrow 0$ in the Riesz space weak topology induced on $V$ by $M$. On the other hand, $\left|F_{y z} f_{m}\right|=\left|\int_{y}^{z} \sin (2 \pi m x) d x\right| \leqq 1 / \pi m$ for all $m, y, z$. Thus $f_{m} \rightarrow 0$ in the weak topology induced on $V$ by $M$. Hence, in this case, the topologies differ.)

1.13. Lemma. Let $M$ be a set of positive functionals on $V$. Suppose $F_{1}$, $F_{2} \in M$ and $\alpha>0$ imply $F_{1}+F_{2} \in M$ and $\alpha F_{1} \in M$. If $U$ is a neighborhood of 0 in the Riesz space weak topology induced on $V$ by $M$, there is an $F \in M$ such that $F|f| \leqq 1$ implies $f \in U$.

Proof. Clearly there are $F_{1}, \cdots, F_{n} \in M$ such that $F_{i}|f| \leqq 1$ for all $i$ implies $f \in U$. Set $F=F_{1}+\cdots+F_{n}$. Then $F \in M$ and for each $i$ we have $F_{i}|f| \leqq F_{1}|f|+\cdots+F_{n}|f|=F|f|$. Thus $F|f| \leqq 1$ implies $f \in U$.

We recall that $M \subset V$ is called a lattice ideal when:

(1) $M$ is a linear subspace of $V$, and

(2) If $F \in M, G \in V$ and $|G| \leqq|F|$, then $G \in M$.

1.14. Theorem. Let $V$ be a Riesz space. In order that there exist a topology on $V$ compatible with the Riesz space structure of $V$ and such that $M$ is the set of all continuous linear functionals on $V$, it is necessary and sufficient that $M$ 
be a lattice ideal in the space of all relatively bounded functionals on $V$.

Proof. Necessity. Suppose the topology exists. Let $F \in M, G$ be relatively bounded and $|G| \leqq|F|$. Since $F \in M, F$ is continuous. Hence $|F|$ is continuous by Lemma 1.9. Since $0 \leqq G^{+} \leqq|G| \leqq|F|$ and $0 \leqq G^{-} \leqq|G| \leqq|F|, G^{+}$and $G^{-}$are continuous by Corollary 1.5. Therefore $G$ is continuous.

Sufficiency. Suppose the condition is satisfied. We consider the Riesz space weak topology induced on $V$ by the positive elements of $M$. Suppose $F \in M$. Then $F^{+}, F^{-} \in M$ since $\left|F^{+}\right| \leqq|F|$ and $\left|F^{-}\right| \leqq|F|$. Thus $F^{+}, F^{-}$are continuous since they belong to $M$ and are positive. Hence $F$ is continuous.

Now suppose that $G$ is a positive continuous functional on $V$. We seek to show $G \in M$. Since $G$ is continuous, the set of $f$ with $|G f| \leqq 1$ is a neighborhood of 0 . Thus, by the lemma, there is an $F \in M$ such that $F|f| \leqq 1$ implies $|G f| \leqq 1$. Consider any fixed positive $f$. For every $\alpha>F f$ we have $F|f / \alpha| \leqq 1$ since $\alpha>0$ and $f \geqq 0$. Thus $|G(f / \alpha)| \leqq 1$; hence $G f=|G f| \leqq \alpha$. Since this inequality holds for all $\alpha>F f, G f \leqq F f$. Since this last inequality holds for all positive $f, G \leqq F$; i.e. $|G| \leqq|F|$. Therefore $G \in M$.

Now let $G$ be an arbitrary continuous functional on $V$. Then $G^{+}$and $G^{-}$ are continuous. By the previous paragraph $G^{+}, G^{-} \in M$. Hence $G \in M$. Thus there is a topology compatible with the Riesz space structure of $V$ and such that the functionals in $M$ are the only continuous linear functionals.

1.15. CoRollary. There is a topology on V, compatible with the Riesz space structure of $V$, such that every relatively bounded linear functional is continuous.

Let $V$ be a topological Riesz space and $V^{*}$ be the set of all continuous linear functionals on $V$. The Riesz space weak topology induced on $V^{*}$ by the positive elements of $V$ will be called the Riesz space weak* topology on $V^{*}$. (We note that a positive element of $V$ is positive when regarded as a functional on $V^{*}$; however an element of $V$ may be positive as a functional on $V^{*}$ without being a positive element of $V$.)

1.16. Theorem. $V^{*}$ endowed with the Riesz space weak* topology is a topological Riesz space.

Proof. We have already shown (Theorem 1.10 ) that $V^{*}$ is a Riesz space and (Lemma 1.11) that the Riesz space weak* topology is compatible with its Riesz space structure.

We next consider the strong topology on $V^{*}$.

1.17. Lemma. Let $A$ be a topologically bounded subset of $V$. Let $B=\left\{f^{+} \mid f \in A\right\}$. Then $B$ is topologically bounded.

Proof. A necessary and sufficient condition for a subset $C$ of $V$ to be bounded is that for every sequence $\left\{f_{n}\right\}$ of elements of $C$, and every sequence $\left\{\alpha_{n}\right\}$ of positive real numbers converging to 0 , the sequence $\left\{\alpha_{n} f_{n}\right\}$ converge to $0\left[4\right.$, vol. 2, p. 6]. Let $\left\{f_{n}^{+}\right\}$be any sequence of elements of $B$ with $f_{n} \in A$. 
Let $\left\{\alpha_{n}\right\}$ be any sequence of positive real numbers converging to 0 . Then $\alpha_{n} f_{n} \rightarrow 0$. Since the mapping $f \rightarrow f^{+}$is continuous, $\alpha_{n} f_{n}^{+}=\left(\alpha_{n} f_{n}\right)^{+} \rightarrow 0$. Therefore $B$ is bounded.

1.18. Theorem. The strong dual of a topological Riesz space is a topological Riesz space. I.e. the strong topology on the set of continuous functionals is compatible with this set's Riesz space structure.

Proof. We have shown that the continuous linear functionals form a Riesz space. We note that the polars of the topologically bounded sets of $V$ form a fundamental system of neighborhoods of 0 relative to the strong topology.

We verify Axiom 3 first. Let $U$ be any strong neighborhood of 0 . Then $U$ contains a neighborhood $W$ of 0 such that $W$ is the polar of a bounded set $A$ of $V$. I.e. $W$ consists of all $F$ such that $F f \leqq 1$ for all $f \in A$. Let $B=\left\{f^{+} \mid f \in A\right\}$. Then $B$ is bounded; hence the polar $U^{\prime}$ of $B$ is a neighborhood of 0 . Now suppose $0 \leqq G \leqq F$ and $F \in U^{\prime}$. Then $F f \leqq 1$ for all $f \in B$. We have $G f=G f^{+}$ $-G f^{-} \leqq G f^{+} \leqq F f^{+} \leqq 1$ for all $f \in A$. Hence $G \in W \subset U$. Thus we have found a $U^{\prime}$ of the required kind.

We now verify Axiom 2. Starting with $U$, any neighborhood of 0 , we find $W, A$ and $B$ as before. Let $C$ be the set of all $g$ for which an $f \in B$ exists with $0 \leqq g \leqq f$. It is clear, since Axiom 3 holds for $V$, that $C$ is bounded. Let $U^{\prime}$ be the polar of $C$. Then $F g \leqq 1$ for all $F \in U^{\prime}, g \in C$. Let $F \in U^{\prime}$ and $f \in A$. Then $f^{+} \in B$ and $0 \leqq g \leqq f^{+}$implies $g \in C$. We have $F^{+} f=F^{+} f^{+}-F^{+} f^{-} \leqq F^{+} f^{+}$ $=1$.u.b. $0 \leq 0 \leq f^{+} F g \leqq 1$. Thus $F^{+} \in W$, the polar of $A$. Since $W \subset U$, we have shown that for every $F \in U^{\prime}, F^{+} \in U$. Thus the mapping $F \rightarrow F^{+}$is continuous at 0 .

2. Projections on Riesz spaces. We now consider, from an algebraic point of view, certain projections on a Riesz space. It is clear that these projections are precisely the ones which correspond to direct sum decompositions of the Riesz space. By such a decomposition we mean a decomposition in the sense of linear spaces with the property that an element is positive if and only if its components are both positive. We note that, in the case where the Riesz space is endowed with a compatible topology, every algebraic direct sum decomposition is a topological direct sum decomposition (Corollary 1.6).

Let $V^{*}$ be a Riesz space. (For example, $V^{*}$ may consist of all continuous linear functionals on a topological Riesz space $V$. In that case $V^{*}$ is completely reticulated.) By an allowable projection we shall mean an idempotent linear mapping $P$ of $V^{*}$ into itself such that both $P$ and $I-P$ are positive. We note that if $P$ is an allowable projection, so is $I-P$. We shall write $R(P)$ for the range of $P$. The following theorem shows, among other things, that an allowable projection is uniquely determined by its range.

2.1. Theorem. Let $P$ be an allowable projection. Then:

(a) Let $F \geqq 0$. Then $P F=0$ if and only if $F$ has the property that whenever 
$G \geqq 0$ is such that $P G=G$ and $F \geqq G$, then $G=0$.

(b) Let $F \geqq 0$. Then $P F=F$ if and only if $F$ has the property that whenever $G \geqq 0$ is such that $P G=0$ and $F \geqq G$, then $G=0$.

(c) Let $P^{\prime}$ be an allowable projection. If $R\left(P^{\prime}\right)=R(P)$, then $P^{\prime}=P$.

(d) Let $P^{\prime \prime}$ be an allowable projection. If $P^{\prime \prime}$ is 0 for the same $F$ as $P$ is 0 , then $P^{\prime \prime}=P$.

Proof. See [7, pp. 471-472].

2.2. Corollary. If $P$ is an allowable projection, $F \in R(P)$ and $0 \leqq G \leqq F$, then $G \in R(P)$.

2.3. Corollary. If $P$ is an allowable projection and $F \in R(P)$, then $F^{+} \in R(P)$ and $F^{-} \in R(P)$.

Proof. We have $0 \leqq F^{+} \leqq F^{+}+F^{-}=|F|=|P F|=\left|P F^{+}-P F^{-}\right| \leqq P F^{+}$ $+P F^{-} \in R(P)$. Hence $F^{+} \in R(P)$. Similarly, $F^{-} \in R(P)$.

2.4. Corollary. If $P_{1}, P_{2}$ are allowable projections and $F \in R\left(P_{1}\right)$, then $P_{2} F \in R\left(P_{1}\right)$.

Proof. Since $F \in R\left(P_{1}\right), F^{+} \in R\left(P_{1}\right)$ and $F^{-} \in R\left(P_{1}\right)$. Since $0 \leqq P_{2} F^{+} \leqq F^{+}$ and $0 \leqq P_{2} F^{-} \leqq F^{-}, P_{2} F^{+} \in R\left(P_{1}\right)$ and $P_{2} F^{-} \in R\left(P_{1}\right)$. Hence $P_{2} F=P_{2} F^{+}$ $-P_{2} F^{-} \in R\left(P_{1}\right)$.

2.5. Theorem. Let $P_{1}, P_{2}$ be allowable projections. Then $P_{1} \leqq P_{2}$ (i.e. $P_{1} F \leqq P_{2} F$ for all positive $\left.F \in V^{*}\right)$ if and only if $R\left(P_{1}\right) \subset R\left(P_{2}\right)$.

Proof. Suppose $P_{1} \leqq P_{2}$. Then $I-P_{1} \geqq I-P_{2}$. Thus if $F \geqq 0$ and $F \in R\left(P_{1}\right)$, $0 \leqq\left(I-P_{2}\right) F \leqq\left(I-P_{1}\right) F=0$; i.e. $F \in R\left(P_{2}\right)$. If $F \in R\left(P_{1}\right)$ is arbitrary, $F^{+}$ $\in R\left(P_{1}\right)$ and $F^{\llcorner} \in R\left(P_{1}\right)$. Hence $F \in R\left(P_{2}\right)$.

Now suppose $R\left(P_{1}\right) \subset R\left(P_{2}\right)$. For any $F \geqq 0$, we have $P_{2} F \geqq P_{2} P_{1} F=P_{1} F$ since $P_{1} F \in R\left(P_{1}\right) \subset R\left(P_{2}\right)$. Thus $P_{2} \geqq P_{1}$.

2.6. Theorem. Let $P_{1}, P_{2}$ be allowable projections. Then $P_{1} P_{2}$ is an allowable projection and $R\left(P_{1} P_{2}\right)=R\left(P_{1}\right) \cap R\left(P_{2}\right)$.

Proof. If $F \in R\left(P_{1}\right) \cap R\left(P_{2}\right), P_{1} P_{2} F=P_{1} F=F$. For any $F \in V^{*}, P_{2} F$ $\in R\left(P_{2}\right)$; hence $P_{1} P_{2} F \in R\left(P_{1}\right) \cap R\left(P_{2}\right)$. It follows that $P_{1} P_{2}$ is idempotent and that $P_{1} P_{2} F=F$ if and only if $F \in R\left(P_{1}\right) \cap R\left(P_{2}\right)$. Clearly, $P_{1} P_{2}$ is positive and linear. For any $F \geqq 0, P_{1} P_{2} F \leqq P_{2} F \leqq F$ since $I-P_{1}$ and $I-P_{2}$ are positive. Thus $I-P_{1} P_{2}$ is positive. Hence $P_{1} P_{2}$ has the required properties.

\subsection{Corollary. Any two allowable projections commute.}

2.8. Theorem. The set of all allowable projections on $V^{*}$ is a lattice with $P_{1} \wedge P_{2}=P_{1} P_{2}$ and $P_{1} \vee P_{2}=P_{1}+P_{2}-P_{1} P_{2}$.

Proof. We note $\left(I-P_{1}\right)\left(I-P_{2}\right)=I-\left(P_{1}+P_{2}-P_{1} P_{2}\right)$. The theorem is then obvious since the three statements $Q_{1} \leqq Q_{2}, R\left(Q_{1}\right) \subset R\left(Q_{2}\right)$ and $I-Q_{1} \geqq I-Q_{2}$ 
are equivalent if $Q_{1}$ and $Q_{2}$ are any allowable projections.

Riesz [10, p. 182], proved the following theorem: Let $M$ be a set of linear functionals on a Riesz space $V$. Suppose whenever $F_{1}, F_{2} \in M$, there is an $F \in M$ such that $F \leqq F_{1}, F \leqq F_{2}$. Then for every positive $f \in V$ :

$$
\text { (g.l.b. } M) f=\underset{F \in M}{\text { g.l.b. }} F f \text {. }
$$

(We note that this theorem can be proved by the method which will be used to prove Theorem 2.9.)

2.9. Theorem. Let $V^{*}$ consist of all continuous linear functionals on $V, a$ topological Riesz space. Let $\odot$ be any nonempty set of allowable projections on $V^{*}$. Then 8 has a g.l.b. $P$ (in the set of all allowable projections). For every positive $F \in V^{*}$ and every positive $f \in V$ :

$$
P F f=\underset{Q \in Q}{g .1 . b .} Q F f
$$

where $Q$ is the set of all finite products of projections in $\mathcal{P}$. Also $R(P)=\bigcap_{Q \in \mathcal{P}} R(Q)$.

Proof. We first note that for each positive $F \in V^{*}, Q F \geqq 0$ for all $Q \in Q$. Thus we may set $P F=$ g.l.b. $Q \in Q Q Q F$ for each positive $F$. Clearly $P F \in V^{*}$ since $0 \leqq P F \leqq F$ (Corollary 1.5). In this way we define a mapping $P$ of the set of positive elements of $V^{*}$ into itself.

Before establishing the linearity of $P$, we note that $P F f=$ g.l.b. $Q \in \mathcal{Q} Q F f$ for each $F \geqq 0$ and $f \geqq 0$. This statement follows from the theorem of Riesz above; for if $Q_{1} F, Q_{2} F \in\{Q F \mid Q \in Q\}$; then $Q_{1} Q_{2} \leqq Q_{1} F, Q_{1} Q_{2} F \leqq Q_{2} F$ and $Q_{1} Q_{2} F \in\{Q F \mid Q \in Q\}$.

We now prove the linearity of $P$. It is clear that $P(\alpha F)=\alpha P F$ for all $\alpha \geqq 0$ and $F \geqq 0$. Let $F_{1}, F_{2} \geqq 0$ be given. Let $f \geqq 0$ and $\epsilon>0$ be given. We can find $Q_{1}, Q_{2} \in Q$ such that $P F_{1} f \geqq Q_{1} F_{1} f-\epsilon$ and $P F_{2} f \geqq Q_{2} F_{2} f-\epsilon$. Since $Q_{1} Q_{2}$ $\leqq Q_{1}$ and $Q_{1} Q_{2} \leqq Q_{2}, P F_{1} f \geqq Q_{1} Q_{2} F_{1} f-\epsilon$ and $P F_{2} f \geqq Q_{1} Q_{2} F_{2} f-\epsilon$. Thus $P F_{1} f$ $+P F_{2} f \geqq Q_{1} Q_{2} F_{1} f+Q_{1} Q_{2} F_{2} f-2 \epsilon=Q_{1} Q_{2}\left(F_{1}+F_{2}\right) f-2 \epsilon$. Since $Q_{1} Q_{2} \in Q$, $P\left(F_{1}+F_{2}\right) f \leqq Q_{1} Q_{2}\left(F_{1}+F_{2}\right) f \leqq P F_{1} f+P F_{2} f+2 \epsilon$. Since $\epsilon$ is arbitrary, $P\left(F_{1}+F_{2}\right) f \leqq P F_{1} f+P F_{2} f$. Now we note that

$$
\begin{aligned}
P\left(F_{1}+F_{2}\right) f & =\underset{Q \in Q}{\text { g.l.b. }}\left(Q F_{1} f+Q F_{2} f\right) \geqq \underset{Q \in Q}{\text { g.l.b. }} Q F_{1} f+\underset{Q \in Q}{\text { g.l.b. }} Q F_{2} f \\
& =P F_{1} f+P F_{2} f .
\end{aligned}
$$

Joining this inequality to the previous one, we have $P\left(F_{1}+F_{2}\right) f=P F_{1} f$ $+P F_{2} f$. Since $f$ is arbitrary, $P\left(F_{1}+F_{2}\right)=P F_{1}+P F_{2}$.

Thus we may extend $P$ to be a linear mapping of $V^{*}$ into itself. Clearly $P$, as extended, is positive and $I-P$ is positive.

Suppose that $F \in \bigcap_{Q \in \mathcal{P}} R(Q)$ is positive. Then $Q F=F$ for each $Q \in \mathcal{P}$, and hence for each $Q \in Q$. Thus $P F=F$. Hence if $F \in \bigcap_{Q \in \mathcal{P}} R(Q)$ is arbitrary, $P F=F$. For any $F \geqq 0$ and $Q \in \mathcal{P}$, we have for each $f \geqq 0,0 \leqq P F f \leqq Q F f$ since 
$Q \in Q$. Thus $0 \leqq P F \leqq Q F$. Since $Q F \in R(Q), P F \in R(Q)$. Since this statement holds for any $Q \in \mathcal{P}, P F \in \cap_{Q \in \mathcal{P}} R(Q)$. It follows that $P^{2} F=P F$ for all $F$. It also follows that $R(P)=\bigcap_{\boldsymbol{Q} \in \mathcal{P}} R(Q)$. Hence $P$ is a g.l.b. for $\mathcal{P}$.

2.10. Corollary. Let $V^{*}$ and $\odot$ be as above. Then $\odot$ has a l.u.b. P. For every positive $F \in V^{*}$ and positive $f \in V$ :

$$
P F f=\underset{Q \in Q}{\text { l.u.b. }} Q F f
$$

where $Q$ consists of all projections of the form $I-\left(I-P_{1}\right) \cdots\left(I-P_{n}\right)$ with $P_{1}, \cdots, P_{n} \in \odot$.

Proof. We apply the above theorem to the set of $I-Q$ with $Q \in \mathcal{P}$.

The following theorem tells whether a given subset of $V^{*}$ is the range of a projection. Before stating the theorem, we recall that a subset $M$ of a completely reticulated Riesz space $V^{*}$ is called a band if:

(a) It is a lattice ideal, and

(b) Whenever $M^{\prime}$ is a subset of $M$ which is majorized in $V^{*}$, the l.u.b. of $M^{\prime}$ is in $M$.

2.11. Theorem. Let $V^{*}$ be a completely reticulated Riesz space. Let $M \subset V^{*}$. There is an allowable projection $P$ with $R(P)=M$ if and only if $M$ is a band.

Proof. We see in the usual way that the existence of a projection with range $M$ is equivalent to the existence of a direct sum decomposition of $V^{*}$ with $M$ for one summand. It follows from a theorem of Riesz that such a decomposition exists if and only if $M$ is a band [10, p. 185].

3. Topologies in relation to bands and projections. In this section, we first characterize the bands in the dual of a topological Riesz space by proving that the bands are precisely the closed ideals. We then consider whether it is possible to decompose every relatively bounded linear functional into a continuous and an "anti-continuous" part; i.e. whether there exists a projection of the set of relatively bounded functionals onto the set of continuous ones. More generally, we determine (see Theorem 3.11) when there exists a projection of the functionals which are continuous in one topology onto those which are also continuous in a second topology. The results of this section are stated in terms of topologies and filters; those of the next section are stated, somewhat more simply, in terms of directed sequences.

Throughout the following discussion $V$ is a Riesz space, with a compatible topology, and $V^{*}$ is the set of all continuous linear functionals on $V$. We note that a suitable choice of topology for $V$ will make $V^{*}$ the set of all relatively bounded linear functionals on $V$ (Corollary 1.15). We first characterize the bands in $V^{*}$.

3.1. Lemma. Let $M \subset V^{*}$ have least upper bound $F \in V^{*}$. Suppose whenever 
$G, H \in M$ there is a $K \in M$ such that $K \geqq G, K \geqq H$. Then $F$ is in the closure $\bar{M}$ of $M$ in the Riesz space weak* topology on $V^{*}$.

Proof. Let $U$ be any Riesz space weak* neighborhood of $F$. Then there is a positive $f \in V$ such that every $G$ for which $|G-F| f \leqq 1$ is in $U$ (Lemma 1.13). Since $F$ is the 1.u.b. of $M$, we know from a theorem of Riesz stated above that $F f=1$.u.b. $G \in M$ Mf. Thus we can find a $G \in M$ such that $(F-G) f \leqq 1$. Since $F \geqq G, F-G \geqq 0$; thus $|G-F| f \leqq 1$. Hence $G \in U$ as well as $G \in M$; i.e. $U \cap M \neq \varnothing$. Since every neighborhood of $F$ meets $M, F \in \bar{M}$.

3.2. Lemma. Let $B$ be $a$ band in $V^{*}$. Then $B$ is closed in the Riesz space weak* topology on $V^{*}$.

Proof. Since $B$ is a band, there is a projection $P$ of $V^{*}$ onto $B$ such that $P$ and $I-P$ are positive (Theorem 2.11). $P$ is continuous by Corollary 1.6. Hence $B$ is closed since it is the inverse image of 0 under the continuous mapping $I-P$.

3.3. Theorem. Let $B \subset V^{*} . B$ is a band in $V^{*}$ if and only if $B$ is a lattice ideal closed in the Riesz space weak* topology on $V^{*}$.

Proof. If $B$ is a band, we see from the definition of a band and the previous lemma that $B$ is a closed ideal.

Conversely, suppose $B$ is a closed ideal. Let $M \subset B$ be bounded from above in $V^{*}$. If we show that the least upper bound $F$ of $M$ is in $B$, the theorem will be proved. Let $M^{\prime}$ be the set of all least upper bounds of finite subsets of $M$. Clearly $F$ is the l.u.b. of $M^{\prime}$ as well as of $M$. Also clearly, $M^{\prime}$ has the property that $G \bigvee H \in M^{\prime}$ whenever $G, H \in M^{\prime}$. Thus by Lemma 3.1, $F \in \bar{M}^{\prime}$. But $M^{\prime} \subset B$ and $B$ is closed; hence $F \in B$ and the theorem is proved.

A filter on $V$ will be called bounded from above if it contains a set $U$ such that there is an $f \in V$ with $U \leqq f$.

3.4. Lemma. Let $B$ be a band in $V^{*}$. Let $F \in V^{*}$ be positive. Let $U$ be the set of $f \in V$ such that $F f \leqq 1$. Suppose $U$ belongs to every filter on $V$ bounded from above which converges to 0 in the Riesz space weak topology induced on $V$ by the positive elements of $B$. Then $F \in B$.

Proof. We prove the lemma by finding, for each positive $f \in V$, a functional $G_{f} \in B$ such that $F \geqq G_{f}$ but $F f \leqq G_{f} f+1$. Then we shall see that $F$ belongs to the closure of the set of $G_{f}$; hence $F$ belongs to $B$.

For each positive $G \in B$, let $U_{G}$ denote the set of $f \in V$ such that $G|f| \leqq 1$. Then the set of all $U_{G}$ is a fundamental system of neighborhoods (i.e. a base for the filter of neighborhoods) of 0 in the Riesz space weak topology induced on $V$ by the positive elements of $B$ (Lemma 1.13).

Let $f \in V$ be fixed and positive. Let $U_{G}^{\prime}$ be the set of elements of $U_{G}$ which are $\leqq f$. Since $\left\{U_{G}\right\}$ is a fundamental system of neighborhoods of 0 , the filter generated by $\left\{U_{a}^{\prime}\right\}$ converges to 0 . Since this filter is bounded from 
above, it contains $U$; i.e. $U$ contains one of the sets $U_{G}^{\prime}$ since these sets form a base for the filter. In other words, there is a positive $F_{f} \in B$ such that $F g \leqq 1$ whenever $F_{f}|g| \leqq 1$ and $g \leqq f$.

Now consider any $g$ with $0 \leqq g \leqq f$. We distinguish the cases $F_{f} g \geqq 1$ and $F_{f} g<1$. Suppose first $F_{f} g \geqq 1$. Then $F_{f}\left(g / F_{f} g\right)=1$. Since $0 \leqq g / F_{f} g \leqq g \leqq f$, we see from the preceding paragraph that $F\left(g / F_{f} g\right) \leqq 1$. Thus in this case $F g \leqq F_{f} g$. Now consider the case where $F_{f} g<1$. By the preceding paragraph, in this case $F g \leqq 1$. Combining our results, we see that in either case $F g$ $\leqq F_{f} g+1$.

Let $G_{f}=F \wedge F_{f}$. Since $\left|G_{f}\right|=F \wedge F_{f} \leqq F_{f} \in B$ and $B$ is a band, $G_{f} \in B$. We note $\left|G_{f}-F\right|=F-G_{f}=F-F \wedge F_{f}=\left(F-F_{f}\right)^{+}$. We have $\left(F-F_{f}\right) g \leqq 1$ for all $g$ with $0 \leqq g \leqq f$ by the preceding paragraph. Hence we have $\left(F-F_{f}\right)+f$ $=1$.u.b.0 $0 \leq f\left(F-F_{f}\right) g \leqq 1$. Thus $\left|G_{f}-F\right| f=\left(F-F_{f}\right)+f \leqq 1$.

Now let $f$ vary. Let $A$ be the set of all $G_{f}$ as $f$ ranges through all the positive elements of $V$. Let $W$ be any Riesz space weak* neighborhood of $F$. We shall show $W \cap A \neq \varnothing$; hence $F \in \bar{A}$. Since $W-F$ is a neighborhood of 0 , there is a positive $f \in V$ such that $|G-F| f \leqq 1$ implies $G \in W$ (Lemma 1.13). By the preceding paragraph, $\left|G_{f}-F\right| f \leqq 1$. Hence $G_{f} \in W$ as well as $A$. Thus $A \cap W \neq \varnothing$. Since $B$ is closed, we have $F \in \bar{A} \subset B$.

Let the topology on $V$ be called $\Im_{1}$. We also consider $\Im_{2}$, a second topology on $V$ compatible with the Riesz space structure of $V$. Let $V_{J_{i}}^{*}$ be the set of $\jmath_{i}$-continuous linear functionals on $V(i=1,2)$. We note that a functional $F$ belongs to $V_{J_{1}}^{*} \cap V_{J_{2}}^{*}$ if and only if it is continuous in the Riesz space weak topology $J_{12}$ induced on $V$ by the positive elements of $V_{J_{1}}^{*} \cap V_{J_{2}}^{*}$ (Theorem 1.14). Thus $F \in V_{J_{1}}^{*} \cap V_{J_{2}}^{*}$ if and only if $U=\{f \mid f \in V, F f \leqq 1\}$ is a $\Im_{12}$-neighborhood of 0 ; i.e. if and only if $U$ belongs to every filter on $V$ which $J_{12}$-converges to 0 . In this way, these filters determine which functionals are in both $V_{\mathfrak{I}_{1}}^{*}$ and $V_{J_{2}}^{*}$; in other words, which functionals are both $J_{1}$ - and $J_{2}$-continuous. In some cases it is true that those of the filters which are bounded from above suffice to determine whether a $I_{1}$-continuous functional is also $J_{2}$ continuous. More precisely, suppose there is a projection $P$, and $P$ and $I-P$ positive, of $V_{J_{1}}^{*}$ onto $V_{J_{1}}^{*} \cap V_{J_{2}}^{*}$; i.e. suppose $V_{J_{1}}^{*} \cap V_{J_{2}}^{*}$ is a band in $V_{J_{1}}^{*}$. It follows from the preceding lemma that the ordered pair $\left(J_{1}, J_{2}\right)$ of topologies satisfies the following axiom:

Axıom $\mathrm{B}_{1}$. If $F \in V_{\Im_{1}}^{*}$ is positive, $U=\{f \mid f \in V, F f \leqq 1\}$, and $U$ belongs to every filter on $V$ bounded from above which is $I_{12}$-convergent to 0 ; then $F$ is $J_{2}$-continuous.

We now consider the converse situation where suitable topologies are given and the existence of a projection is to be established. Again $V$ is a Riesz space with a compatible topology. By $V_{R}^{*}$ we mean the set of all relatively bounded linear functionals on $V$. If $f \in V$, by $V(f)$ we mean the set of all open neighborhoods of $f$. If $U \subset V$, by $U^{+}$we mean the set of all positive elements in $U$. 
We shall define a mapping $T$ of $V_{R}^{*}$ into itself. Let $F \in V_{R}^{*}$ be fixed and positive. Let $f \in V$ be positive. We write:

$$
G f=\operatorname{l.u}_{U \in \mathcal{U}(j)}\left(\underset{g \in U^{+}}{\text {g.l.b. }} F g\right) .
$$

We note that, for each $U, 0 \leqq$ g.l.b. ${ }_{g \in U^{+}} F g \leqq F f$ since $f \in U^{+}$. Thus $0 \leqq G f \leqq F f$.

3.5. Lemma. The functional $G$ is additive and positively homogeneous on the positive elements of $V$.

Proof. Let $f, g \geqq 0$ be given. Let $\epsilon>0$ be given. Let $\alpha=G f+\epsilon / 2, \beta=G g$ $+\epsilon / 2$. Pick $U \in U(f+g)$. There are neighborhoods $U_{1}, U_{2}$ of $f, g$ respectively such that $U_{1}+U_{2} \subset U$. The definitions of $\alpha, \beta$ and $G$ imply that there are $f_{1} \in U_{1}^{+}, f_{2} \in U_{2}^{+}$such that $F f_{1}<\alpha, F f_{2}<\beta$. We note $f_{1}+f_{2} \in U^{+}$and $F\left(f_{1}+f_{2}\right)$ $<\alpha+\beta$. Thus g.l.b. $h \in U^{+} F h<a+\beta$. Since this inequality holds for any $U \in \mathcal{V}(f+g), \quad G(f+g) \leqq \alpha+\beta=G f+G g+\epsilon$. Since $\epsilon$ is arbitrary, $G(f+g)$ $\leqq G f+G g$.

Now consider the mappings $\phi(h)=h \wedge f$ and $\psi(h)=h-h \wedge f$. We note both $\phi$ and $\psi$ are continuous, $\phi(h)+\psi(h)=h, \phi(f+g)=f$ and $\psi(f+g)=g$. For any $U_{1} \in \mathcal{V}(f)$ and $U_{2} \in \mathcal{V}(g)$ we can find $W_{1}, W_{2} \in \mathcal{V}(f+g)$ such that $\phi\left(W_{1}\right) \subset U_{1}$ and $\psi\left(W_{2}\right) \subset U_{2}$. Let $W=W_{1} \cap W_{2}$. Then $W \in \mathcal{V}(f+g), \phi(W) \subset U_{1}$ and $\psi(W) \subset U_{2}$. Since $\phi, \psi$ are positive mappings, we have $\phi\left(W^{+}\right) \subset U_{1}^{+}, \psi\left(W^{+}\right)$ $\subset U_{2}^{+}$. We have:

$$
\begin{aligned}
G(f+g) \geqq \underset{h \in W^{+}}{\text {g.l.b. } F h} & =\underset{h \in W^{+}}{g \text { g.l.b. }}\{F[\phi(h)]+F[\psi(h)]\} \\
& \geqq \underset{h \in W^{+}}{\text {g.l.b.b. }} F[\phi(h)]+\underset{h \in \mathbb{W}^{+}}{\operatorname{g.l} . \mathrm{b} .} F[\psi(h)] \\
& \geqq \underset{k \in U_{1}^{+}}{\text {g.l.b.b. }} F k+\underset{k \in U_{2}^{+}}{\text {g.l.b. }} F k .
\end{aligned}
$$

Since this is true for any $U_{1} \in \mathcal{V}(f)$ and $U_{2} \in \mathcal{V}(g), G(f+g) \geqq G f+G g$. Joining this inequality to the previous one, we have $G(f+g)=G f+G g$.

We extend $G$ to all of $V$ by linearity. We note that $G$ as extended is positive and linear. We write $T F=G$; thus $T$ is a mapping of the set of positive functionals into itself.

3.6. Lemma. If $F_{1}, F_{2}$ are positive linear functionals, then $T\left(F_{1}+F_{2}\right)$ $=T F_{1}+T F_{2}$.

Proof. Let $f \geqq 0$ be given. For a given $\epsilon>0$, let $U_{1}, U_{2} \in V(f)$ be such that g.l.b. $\sigma_{\in} U_{1}^{+} F_{1} g>T F_{1} f-\epsilon$ and g.l.b. $\in_{\in} U_{2}^{+} F_{2} g>T F_{2} f-\epsilon$. Let $U=U_{1} \cap U_{2}$. Then

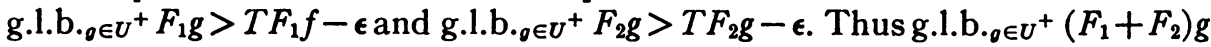
$>T F_{1} f+T F_{2} f-2 \epsilon$. Therefore $T\left(F_{1}+F_{2}\right) f>T F_{1} f+T F_{2} f-2 \epsilon$. Since $\epsilon$ is arbitrary, $T\left(F_{1}+F_{2}\right) f \geqq T F_{1} f+T F_{2} f$.

Now consider the mapping $\Phi(g, h)=g \wedge h$ of $V \times V \rightarrow V$. We note that this 
mapping is continuous and $\Phi(f, f)=f$. Choose any $U \in \mathcal{V}(f)$. Then we can find $U_{1}, U_{2} \in \mathcal{V}(f)$ such that $\Phi\left(U_{1} \times U_{2}\right) \subset U$. We note $T F_{1} f \geqq$ g.l.b. ${ }_{g} \in U_{1}^{+} F_{1} g$, $T F_{2} f \geqq$ g.l.b. ${ }_{g} \in U_{2}^{+} F_{2} h$ and $\Phi\left(U_{1}^{+} \times U_{2}^{+}\right) \subset U^{+}$. Thus

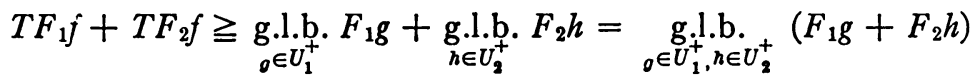

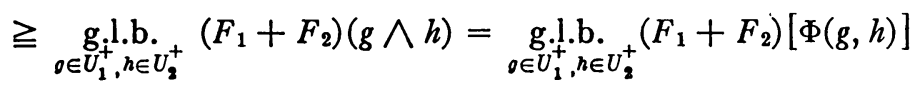

$$
\begin{aligned}
& =\underset{\mathbf{k} \in \Phi\left(U_{1}^{+} \times U_{2}^{+}\right)}{\text {g.l.b. }}\left(F_{1}+F_{2}\right) k \geqq \underset{k \in U^{+}}{\text {g.l.b. }}\left(F_{1}+F_{2}\right) k .
\end{aligned}
$$

Since this inequality holds for all $U \in \mathcal{U}(f), T F_{1} f+T F_{2} f \geqq T\left(F_{1}+F_{2}\right) f$. This inequality and that of the preceding paragraph establish that $T\left(F_{1}+F_{2}\right) f$ $=T F_{1} f+T F_{2} f$ for all $f \geqq 0$, and hence for all $f$.

Thus we may extend $T$ to all of $V_{R}^{*}$ by linearity. $T$, as extended, is a positive linear mapping of $V_{R}^{*}$ into itself. $T$ has the property that $T F \leqq F$ for $F \geqq 0$.

3.7. Lемma. If $F \in V_{R}^{*}$ is continuous, $T F=F$.

Proof. If $F \geqq 0$, it is clear from the definition of $T$ that $T F=F$. For arbitrary $F$, it is enough to note that the continuity of $F$ implies that $F^{+}$and $F^{-}$ are continuous.

3.8. Lemma. $T^{2}=T$.

Proof. Pick $F \in V_{R}^{*}$ and $f \in V$ both positive. We note

$$
T F g=\underset{W \in \mathcal{U}(g)}{\text { l.u.b. }}\left(\underset{h \in W^{+}}{\text {g.l.b. } F h}\right)
$$

for all $g \geqq 0$. Let $U \in \mathcal{V}(f)$ and $g \in U^{+}$. Then $U \in \mathcal{V}(g)$ and thus $T F g$ $\geqq$ g.l.b. $h \in U^{+} F h$. Since this inequality holds for all $g \in U^{+}$, g.l.b. $g \in U^{+} T F g$ $\geqq$ g.l.b. $h \in U^{+} F h$. Since this last inequality holds for all $U \in V(f)$, 1.u.b. $U \in \mathcal{V}(f)\left(\right.$ g.l.b. $\left.{ }_{o \in U^{+}} T F g\right) \geqq$ l.u.b. $U \in \mathcal{V}(f)$ (g.l.b. $\left.h \in U^{+} F h\right)$, i.e. $T(T F) f \geqq T F f$. Since this is true for all $f \geqq 0, T(T F) \geqq T F$. But, since $T F \geqq 0, T(T F) \leqq T F$. Thus $T(T F)=T F$ for all $F \geqq 0$, and hence for all $F \in V_{R}^{*}$.

Now suppose that $V$ is endowed with two compatible topologies $J_{1}, J_{2}$. $J_{2}$ will play the role of the topology used in the preceding paragraphs. Let $V_{\mathfrak{J}_{i}}^{*}$ be the set of $\mathfrak{I}_{i}$-continuous linear functionals on $V(i=1,2)$. The preceding discussion shows that there is a projection $T$ on $V_{R}^{*}$ such that: (a) $T$ and $I-T$ are positive, and (b) $T F=F$ if $F$ is $J_{2}$-continuous. We note $|T F|$ $=\left|T F^{+}-T F^{-}\right| \leqq\left|T F^{+}\right|+\left|T F^{-}\right|=T F^{+}+T F^{-} \leqq F^{+}+F^{-}=|F|$. Thus, since by Theorem $1.14 V_{J_{1}}^{*}$ is an ideal in $V_{R}^{*}, T$ maps $V_{J_{1}}^{*}$ into itself. Therefore the restriction of $T$ to $V_{J_{1}}^{*}$ is a projection on $V_{\mathfrak{J}_{1}}^{*}$ and has the properties (a) and (b) just mentioned. From now on, $T$ stands for this restriction.

We consider the following axiom, which the ordered pair $\left(J_{1}, J_{2}\right)$ may satisfy: 
Axıом $\mathrm{B}_{2}$. If $F \in V_{J_{1}}^{*}$ is positive, $U=\{f \mid f \in V, F f \leqq 1\}$, and $U$ belongs to every filter bounded from above which is $J_{2}$-convergent to 0 ; then $F$ is $I_{2}$ continuous.

We note that Axiom $B_{2}$ implies Axiom $B_{1}$, which was previously stated.

3.9. Lemma. Suppose Axiom $\mathrm{B}_{2}$ is satisfied. Then if $F \in V_{\mathfrak{J}_{1}}^{*}$ is positive and $T F=F, F$ is $\mathfrak{J}_{2}$-continuous.

Proof. Let $U=\{f \mid f \in V, F f \leqq 1\}$. It is enough to show that $U$ belongs to every filter bounded from above which is $J_{2}$-convergent to 0 . Let $X$ be such a filter. Since $X$ is bounded from above, there are an $X \in X$ and an $h \in V$ such that $X \leqq h$. We note $h \geqq 0$.

We have, using the definition of $T$,

$$
\begin{aligned}
0 & =F(-h)+F(h)=F(-h)+T F h=F(-h)+\underset{W \in \mathcal{V}(h)}{1 . u . b} .\left(\underset{k \in W^{+}}{\text {g.l.b.b. }} F k\right) \\
& =\underset{W \in \mathcal{V}(h)}{\text { l.u.b. }}\left(\underset{\boldsymbol{w} \in W^{+}}{\text {g.l.b.b. }} F(k-h)\right) .
\end{aligned}
$$

Thus there is a $W \in \mathcal{V}(h)$ such that g.l.b. $k \in W^{+} F(k-h) \geqq-1$. We note $F(k-h) \geqq-1$ for all $k \in W^{+}$. Let $W^{\prime}=h-W$; then $W^{\prime}$ is a $J_{2}$-neighborhood of 0 . If $f \in W^{\prime}$ and $f \leqq h, h-f \in W^{+}$; thus $F(-f)=F[(h-f)-h] \geqq-1$. Thus there is a $J_{2}$-neighborhood $W^{\prime}$ of 0 such that $F f \leqq 1$ for all $f \in W^{\prime}$ with $f \leqq h$.

Since $X$ is $J_{2}$-convergent to $0, W^{\prime} \in X$. Thus $X \cap W^{\prime} \in X$. If $f \in X \cap W^{\prime}$, both $f \leqq h$ and $f \in W^{\prime}$; thus $F f \leqq 1$. Hence $U \supset X \cap W^{\prime}$. Therefore $U \in X$ and the lemma is proved.

3.10. Lemma. Suppose Axiom $\mathrm{B}_{2}$ is satisfied. Then if $F \in V_{\mathfrak{J}_{1}}^{*}$ and $T F=F$, $F$ is $\mathfrak{J}_{2}$-continuous.

Proof. We have $F=T F=T F^{+}-T F^{-} . T\left(T F^{+}\right)=T F^{+}$and $T\left(T F^{-}\right)=T F^{-}$; thus $T F^{+}$and $T F^{-}$are continuous by the previous lemma.

3.11. Theorem. Let $J_{1}, J_{2}$ be two compatible topologies on a Riesz space $V$. Then a projection $P$ on the set $V_{J_{1}}^{*}$ of $J_{1}$-continuous linear functionals with the properties:

(a) $P$ and $I-P$ are both positive, and

(b) The range of $P$ is the set of functionals both $J_{1}$-continuous and $J_{2}$ continuous,

exists if and only if the ordered pair $\left(J_{1}, J_{2}\right)$ satisfies Axiom $\mathrm{B}_{1}$. If the projection exists, it is unique and it is continuous in any topology on $V_{J_{1}}^{*}$ compatible with the Riesz space structure of $V_{J_{1}}^{*}$.

Proof. Suppose first that $\left(J_{1}, J_{2}\right)$ satisfies Axiom $B_{1}$. Let $J_{12}$ be the Riesz space weak topology induced on $V$ by the postitive functionals in $V_{\mathfrak{J}_{1}}^{*} \cap V_{\mathfrak{J}_{2}}^{*}$. We note by Theorems 1.12 and 1.14 that the set of $J_{12}$-continuous functionals 
is $V_{J_{1}}^{*} \cap V_{J_{2}}^{*}$. Clearly, since $\left(\Im_{1}, J_{2}\right)$ satisfies Axiom $B_{1},\left(J_{1}, J_{12}\right)$ satisfies Axiom $\mathrm{B}_{2}$. Using $J_{12}$ as the topology involved in the construction, construct $T$ in the manner described above; we take this $T$ as $P$. It is clear from the previous discussion that $P$ satisfies (a) and (b).

Now suppose $P$ satisfying (a) and (b) exists. We have previously noted that Axiom $B_{1}$ is satisfied. $P$ is unique by Theorem 2.1 and continuous by Corollary 1.6.

Each of the following theorems involves several topologies on $V . J_{\iota}$, with any index $\iota$, will denote a topology on $V$ compatible with the Riesz space structure of $V ; V_{J_{\mathfrak{l}}}^{*}$ will denote the set of $\mathfrak{J}_{\mathfrak{\imath}}$-continuous linear functionals. $P_{J_{1} \rightarrow J_{2}}$ will stand for the unique projection, of the kind under consideration, of $V_{J_{1}}^{*}$ onto $V_{J_{1}}^{*} \cap V_{J_{2}}^{*}$. $\left(P_{J_{1} \rightarrow J_{2}}\right.$ does not always exist.)

3.12. THEOREM. If $\mathfrak{J}_{2}$ is finer than ${J_{1}}_{1}$ and $P_{J_{2} \rightarrow J_{3}}$ exists, then $P_{\mathfrak{J}_{1} \rightarrow J_{3}}$ exists and is the restriction of $P_{J_{2} \rightarrow J_{3}}$ to $V_{J_{1}}^{*}$.

Proof. It is enough to note that $P_{\Im_{2} \rightarrow J_{3}}$ maps $V_{J_{1}}^{*}$ into itself. We note $|P F|=\left|P F^{+}-P F^{-}\right| \leqq P F^{+}+P F^{-} \leqq F^{+}+F^{-} \leqq|F|$, where $P$ denotes $P_{J_{2} \rightarrow J_{3}}$, for each $F \in V_{J_{1}}^{*}$. Since $V_{J_{1}}^{*}$ is an ideal in $V_{R}^{*}$, the theorem is proved.

3.13. Theorem. If $J_{2}$ is finer than $J_{1}$ and $P_{J_{3} \rightarrow J_{2}}$ and $P_{J_{2} \rightarrow J_{1}}$ exist, then $P_{J_{3} \rightarrow J_{1}}$ exists and $P_{J_{3} \rightarrow J_{1}}=P_{J_{2} \rightarrow J_{1}} P_{J_{3} \rightarrow J_{2}}$.

Proof. The theorem is trivial.

3.14. Theorem. Let $\mathfrak{J}_{0}$ be given. Let $\mathfrak{T}$ be any collection of topologies $\mathfrak{J}_{\mathfrak{}}$ such that $P_{\mathfrak{J}_{0} \rightarrow J}$ exists for each $\mathfrak{J}_{\mathfrak{\imath}} \in \mathfrak{T}$. Then there is a topology $\mathfrak{J}_{1}$ such that:

(a) A linear functional is $J_{1}$-continuous if and only if it is continuous for each $\mathfrak{J}_{\mathrm{b}} \in \mathfrak{T}$, and

(b) $P_{\coprod_{0} \rightarrow J_{1}}$ exists.

Proof. The existence of a topology satisfying (a) is trivial (Theorem 1.14). We shall show that any such topology satisfies (b). Let

$$
P=\underset{J_{\mathfrak{\imath}} \in \mathfrak{I}}{\operatorname{g.l}} P_{\mathfrak{J}_{0} \rightarrow J_{\mathfrak{I}}} \text {. }
$$

By Theorem 2.9, $P$ exists. This theorem also states that the range of $P$ is $\cap_{J_{\mathfrak{\imath}} \in \mathfrak{I}} V_{\mathfrak{J}_{\mathfrak{t}}}^{*}$. Thus $P_{\mathfrak{J}_{0} \rightarrow \mathfrak{I}_{1}}$ exists.

3.15. Theorem. If $P_{\Im_{1}^{i} \rightarrow J_{3}}$ and $P_{\Im_{2} \rightarrow J_{3}}$ both exist, then the restrictions of these projections to $V_{J_{1}}^{*} \cap V_{J_{2}}^{*}$ are equal.

Proof. We apply Theorems 1.14 and 3.12 .

4. Definition of topologies on Riesz spaces by means of sequences. The preceding section dealt with topologies; however the results of Riesz [10, p. 206]; Gordon and Lorch [7, p. 472]; and Bauer [1, p. 106], deal with monotone sequences. (Bauer considered directed sequences.) Each of these 
results may be interpreted as establishing the existence of a projection. In each case, the projection was one mapping a class of linear functionals onto those functionals for which $F f_{\imath} \rightarrow 0$ whenever $\left\{f_{\imath}\right\}_{\iota \in I}$ belongs to a certain set of directed sequences. We determine in Theorems 4.6 and 4.7 exactly when such a projection exists.

Let $V$ be a Riesz space. We are interested in studying the linear functionals $F$ on $V$ which are such that $F f_{\imath} \rightarrow 0$ whenever $\left\{f_{\imath}\right\}_{\iota} \in I$ is one of a designated collection of directed sequences of elements of $V$. (For example, the $a$-integrals of Lorch [8] are characterized in this way.) It will be shown that the relatively bounded functionals with the desired property are precisely those which are continuous in a certain topology, in which $V$ is a topological Riesz space. (If the given collection of sequences does not satisfy the conditions below, it is possible to modify this collection so that it will satisfy the conditions but so that the set of functionals is not changed.)

We consider a set $S$ of directed sequences of positive elements of $V$. The sequences of $\delta$ need not all have the same indexing set. We suppose, for convenience, that:

(1) If $\left\{f_{\imath}\right\}_{\iota \in I} \in \mathcal{S}$ and $\left\{g_{\imath}\right\}_{\iota \in I}$ are such that $g_{\iota} \leqq f_{\imath}$ for all $\iota \in I$, then $\left\{g_{\imath}\right\}_{\imath \in I} \in S$.

(2) For each $f \geqq 0$, the ordinary sequence $(f, f / 2, f / 3, \cdots) \in \mathcal{S}$.

(3) If $\left\{f_{\imath}\right\}_{\iota \in I} \in S$ and $\alpha>0$, then $\left\{\alpha f_{\imath}\right\}_{\iota \in I} \in S$.

We begin by defining a set $v^{+}$of subsets of the set of positive elements of $V$. We define $W \in \mathcal{V}^{+}$if:

(a) For each $\left\{f_{\imath}\right\}_{\iota \in I} \in \mathcal{S}$ there is a $\nu \in I$ such that $\iota \geqq \nu$ implies $f_{\iota} \in W$, and

(b) $W$ is convex.

We note that each element of $v^{+}$contains 0 since $(0,0,0, \cdots) \in \mathcal{S}$. We note next that the intersection of any two sets in $v^{+}$is in $v^{+}$. Also if $W \in V^{+}$and $\alpha>0$, then $\alpha W \in v^{+}$.

We now define a set $v$ of subsets of $V$ by $v=\left\{W-W \mid W \in v^{+}\right\}$. We note that if $U=W-W$ and $W \in \mathcal{V}^{+}, U \supset W$ since $0 \in W$; i.e. each element of $v$ contains an element of $v^{+} . v$ is a filter base; to see this we note that each element of $v$ contains 0 and that $(W-W) \cap\left(W^{\prime}-W^{\prime}\right) \supset W \cap W^{\prime}-W \cap W^{\prime}$ when $W, W^{\prime} \subset V$. Also if $U \in \mathcal{V}$ and $\alpha>0$, then $\alpha U \in v$.

Clearly the elements of $v$ are symmetric and convex; we shall show that they are also absorbent. Let $U \in \mathcal{V}$ and $f \in V$. Then there is a $W \in \mathcal{V}^{+}$such that $U=W-W$. Since $\left(f^{+}, f^{+} / 2, f^{+} / 3, \cdots\right),\left(f^{-}, f^{-} / 2, f^{-} / 3, \cdots\right) \in \mathcal{S}$ and $W \in \mathcal{V}^{+}$, there is a positive integer $n$ such that $f^{+} / n, f^{-} / n \in W$. Thus $f^{+} / n$ $-f^{-} / n=f / n \in U$; hence $U$ is absorbent.

Since the sets in $V$ are convex, absorbent and symmetric, there is a unique topology in which $V$ is a locally convex topological vector space and $v$ is a fundamental system of neighborhoods of 0 [4, vol. 1, p. 57]. Since each element of $v$ contains an element of $v^{+}$, it is clear that the sequences in $S$ con- 
verge to 0 in this topology. From now on, we consider $V$ to be endowed with this topology, and proceed to show that $V$ is a topological Riesz space.

(It is not in general true that $V$, with the topology defined above, is a Hausdorff space. In particular cases, we can show that $V$ is Hausdorff by showing the existence of suitable continuous linear functionals.)

4.1. Lemma. Each neighborhood $U$ of 0 contains a neighborhood $U^{\prime}$ of 0 with the following property: If $f \in U^{\prime}$ and $0 \leqq g \leqq f$, then $g \in U$.

Proof. $U$ contains a neighborhood $U_{1}$ which belongs to $V$ since $U$ is a fundamental system of neighborhoods of 0 . We define $W$, consisting of positive elements of $V$, as follows: $f \in W$ if $f \geqq 0$ and there does not exist a $g$ such that $0 \leqq g \leqq f$ and $g \notin U_{1}$. Clearly if $f \in W$ and $0 \leqq g \leqq f$, then $g \in U_{1}$.

We show that $W$ is convex. Let $f_{1}, f_{2} \in W$ and $f=\alpha f_{1}+\beta f_{2}$ with $\alpha, \beta>0$ and $\alpha+\beta=1$. Suppose $f \notin W$. Then there is a $g \notin U_{1}$ with $0 \leqq g \leqq f$. We note $g=\alpha\left(f_{1} \wedge g / \alpha\right)+\beta\left\{f_{2}-\left[f_{2}-g / \beta+(\alpha / \beta)\left(f_{1} \wedge g / \alpha\right)\right]\right\}$. Let $h=f_{1} \wedge g / \alpha$ and $k=f_{2}$ $-\left[f_{2}-g / \beta+(\alpha / \beta)\left(f_{1} \wedge g / \alpha\right)\right]$. Then $g=\alpha h+\beta k$. Clearly $0 \leqq h \leqq f_{1}$ since $f_{1}$, $g \geqq 0$ and $\alpha>0$. Also $\alpha h=\left(\alpha f_{1}\right) \wedge g \leqq g$. Thus $k=(1 / \beta)(g-\alpha h) \geqq 0$. We note $\beta\left[f_{2}-g / \beta+(\alpha / \beta)\left(f_{1} \wedge g / \alpha\right)\right]=\beta f_{2}-g+\left(\alpha f_{1}\right) \wedge g=\left(\beta f_{2}+\alpha f_{1}\right) \wedge\left(g+\beta f_{2}\right)-g$ $\geqq\left(\beta f_{2}+\alpha f_{1}\right) \wedge g-g=f \wedge g-g=g-g=0$. Thus $k=f_{2}-\left[f_{2}-g / \beta+(\alpha / \beta)\left(f_{1} \wedge g / \alpha\right)\right]$ $\leqq f_{2}$. We have $0 \leqq h \leqq f_{1} \in W$ and $0 \leqq k \leqq f_{2} \in W$. Hence $h, k \in U_{1}$. Since $g=\alpha h$ $+\beta k$ and $U_{1}$ is convex, $g \in U_{1}$. But $g \notin U_{1}$; therefore $f \in W$ and $W$ is convex.

Now suppose $\left\{f_{\imath}\right\}_{\iota \in I} \in \mathcal{S}$. For each $\iota \in I$ such that $f_{\imath} \in W$, we can choose a $g_{\imath} \notin U_{1}$ such that $0 \leqq g_{\imath} \leqq f_{\imath}$. For each $\iota$ such that $f_{\imath} \in W$, let $g_{\imath}=f_{\imath}$. By property (1) of $S,\left\{g_{\imath}\right\}_{\iota \in I} \in S$. Hence, since $U_{1}$ is a neighborhood of 0 and each sequence in $S$ converges to 0 , there is a $\nu$ such that $g_{\iota} \in U_{1}$ for all $\iota \geqq \nu$. Thus for all $\iota \geqq \nu, f_{\iota} \in W$. It follows that $W \in \mathcal{V}^{+}$.

Since $W \in \mathcal{V}^{+}, W-W$ is a neighborhood of 0 . Suppose $f \in W-W$ and $0 \leqq g \leqq f$. Then there are $f_{1}, f_{2} \in W$ such that $f=f_{1}-f_{2}$. We have, since $f_{2} \geqq 0$, $0 \leqq g \leqq f \leqq f_{1} \in W$. Hence $g \in U_{1} \subset U$. Thus $W-W$ is a neighborhood of 0 with the required property.

\subsection{Lemma. The mapping $f \rightarrow f^{+}$of $V$ into itself is continuous at 0.}

Proof. Let $U$ be any neighborhood of 0 . Then, by the previous lemma, there is a $U_{1}$ such that if $0 \leqq g \leqq f \in U_{1}, g \in U$. There is a $U_{2} \in \mathcal{V}$ such that $U_{2} \subset U_{1}$. Let $W \in \mathcal{V}^{+}$be such that $U_{2}=W-W$. Suppose $f \in U_{2}$. To prove the lemma it is enough to show $f^{+} \in U$. Since $f \in U_{2}=W-W$, there are $f_{1}, f_{2} \in W$ such that $f=f_{1}-f_{2}$. Since $f \leqq f_{1}$ and $0 \leqq f_{1}$, we have $0 \leqq f^{+} \leqq f_{1} \in W \subset U_{2} \subset U_{1}$. Hence $f^{+} \in U$.

Thus we have shown that $V$, endowed with the topology defined above, is a topological Riesz space.

4.3. Lemma. Let $F$ be a linear functional on $V$ and $W=\{f \mid f \in V, f \geqq 0$, $|F f| \leqq 1\}$. If $W \in \mathcal{V}^{+}, F$ is continuous. 
Proof. Let $\epsilon>0$ be given. Let $U=(\epsilon / 2)(W-W)$. Clearly $(2 / \epsilon) U \in \mathcal{V}$; hence $U$ is a neighborhood of 0 . Let $f \in U$; then there are $g, h \in W$ such that $f=(\epsilon / 2)(g-h)$. We have $|F f|=|(\epsilon / 2) F(g-h)| \leqq(\epsilon / 2)(|F g|+|F h|)$ $\leqq(\epsilon / 2)(1+1)=\epsilon$. Since this inequality holds for every $f \in U$, a neighborhood of 0 , the lemma is proved.

4.4. Theorem. Let $V$ be a Riesz space and $\mathrm{s}$ be a set of directed sequences of positive elements of $V$ satisfying (1), (2) and (3) above. Then the topology described above is compatible with the Riesz space structure of $V$. Furthermore, a linear functional $F$ on $V$ is continuous if and only if $\left\{F f_{1}\right\}_{\iota} \in I$ converges to 0 whenever $\left\{f_{l}\right\}_{\iota \in I} \in \mathcal{S}$.

Proof. We have already shown that the topology is compatible. Each of the sequences in $\delta$ converges to 0 . Thus if $F$ is continuous, $\left\{F f_{\imath}\right\}_{\iota} \in I$ converges to 0 whenever $\left\{f_{\imath}\right\}_{\iota \in I} \in S$.

Suppose conversely that $\left\{F f_{\imath}\right\}_{\iota \in I}$ converges to 0 whenever $\left\{f_{l}\right\}_{i \in I} \in \mathcal{S}$. Then for each $\left\{f_{\imath}\right\}_{\iota \in I} \in \mathcal{S}$, there is a $\nu$ such that $\left|F f_{\iota}\right| \leqq 1$ when $\iota \geqq \nu$. Let $W=\{f|f \in V, f \geqq 0| F f \mid, \leqq 1\}$; then $f_{\iota} \in W$ when $\iota \geqq \nu$. Since $W$ is clearly convex, the theorem follows from the preceding lemma.

We are interested in the existence of a projection of the kind studied above. For this reason, we state Axiom $B_{3}$ :

Ахıом $\mathrm{B}_{3}$. If $F$ is a positive linear functional on $V, U=\{f \mid f \in V, F f \leqq 1\}$, and $U$ belongs to every filter on $V$ bounded from above which is convergent to 0 ; then $F$ is continuous.

Call the topology we are considering $J_{2}$. Then Axiom $B_{3}$ may be stated in the following alternative form:

Aхгом $B_{3}^{\prime}$. For each compatible topology $J_{1}$ on $V$, the pair $\left(J_{1}, J_{2}\right)$ satisfies Axiom $B_{2}$.

4.5. Lemma. Suppose each of the sequences in $S$ is bounded; i.e. for each $\left\{f_{\imath}\right\}_{\iota} \in I \in S$ there is an $f \in V$ such that $f_{\iota} \leqq f$ for all $\iota \in I$. Then Axiom $\mathbf{B}_{3}$ is satisfied.

Proof. Let $W=\{f \mid f \in U, f \geqq 0\}$. Then $W=\{f \mid f \in V, F f \leqq 1, f \geqq 0\}$ $=\{f|f \in V| F f \mid, \leqq 1, f \geqq 0\}$ since $F$ is positive. By Lemma 4.3, it is enough to show $W \in \mathcal{V}^{+}$. Let $\left\{f_{l}\right\}_{\iota \in I} \in S$; then this sequence is bounded from above. Hence the filter associated with this sequence is bounded from above. This filter converges to 0 since the sequence does. Thus $U$ belongs to the filter; i.e. there is a $\nu \in I$ such that $f_{\iota} \in U$ for all $\iota \geqq \nu$. Since all the $f_{\iota}$ are positive, $f_{\imath} \in W$ for all $\iota \geqq \nu$. Thus, since $W$ is clearly convex, $W \in \mathcal{V}^{+}$, which proves the lemma.

4.6. Theorem. Let $V^{*}$ be the set of continuous functionals on $V$ in any compatible topology. Suppose $S$ consists of bounded sequences. Then there is a projection $P$ (with $P$ and $I-P$ positive) of $V^{*}$ onto the set of $F \in V^{*}$ for which $\left\{f_{\imath}\right\}_{\imath \in I} \in S$ implies $F f_{\imath} \rightarrow 0$. 
Proof. The theorem follows at once from the lemma and Theorem 3.11.

4.7. Theorem. Let $V^{*}$ be the set of continuous functionals on $V$ in any compatible topology. Suppose $P$ is a projection of $V^{*}$ with $P$ and $I-P$ positive. Then there is a collection $S$ of bounded directed sequences such that $F \in V^{*}$ is in the range of $P$ if and only if $F f_{\imath} \rightarrow 0$ for each $\left\{f_{1}\right\}_{\iota} \in I \in S$.

Proof. We apply Theorem 3.11 . Let $J_{1}$ be the given topology on $V$. Let $J_{2}$ be the Riesz space weak topology induced on $V$ by the positive elements in the range of $P$; thus the range of $P$ consists of the $J_{2}$-continuous functionals. By Theorem 3.11, Axiom $B_{1}$ is satisfied. Clearly $J_{12}$, in the notation of the axiom, $=J_{2}$. This topology is the only one to figure in the remainder of the proof.

Let $x$ be a filter bounded from above converging to 0 . Then there is a $Z \in X$ which is bounded from above. Let $X^{\prime}=\{X \mid X \in X, X \subset Z\}$. Then we may regard $x^{\prime}$ as a directed set ordered by inclusion; i.e. $X \geqq Y$ if $X \subset Y$. For each $X \in X^{\prime}$, choose $f_{X} \in X$; thus we obtain a directed sequence $\left\{f_{X}\right\}_{X \in X^{\prime}}$. Clearly this sequence converges to 0 and is bounded from above. Thus $\left\{f_{X}^{+}\right\}_{X \in X^{\prime}}$ converges to 0 and is bounded. We let $\delta$ be the set of all sequences $\left\{f_{X}^{+}\right\}_{X \in X^{\prime}}$ which can be obtained in this way for all choices of $X, Z$ and the $f_{X}$.

Now suppose $F \in V^{*}$ is positive and such that $F f_{X} \rightarrow 0$ for every $\left\{f_{X}\right\}_{\boldsymbol{X}} \in \mathfrak{X}^{\prime}$ $\in \mathcal{~ . ~ L e t ~} U=\{f \mid f \in V, F f \leqq 1\}$. If we show that $U$ belongs to every filter bounded from above which converges to 0 , it will follow from Axiom $B_{1}$ that $F$ is continuous. Let $x$ be bounded from above. Suppose $U \notin x$. Then for each $X \in X, X \nsubseteq U$. Let $x^{\prime}$ be as in the preceding paragraph. For each $X \in X^{\prime}$, we can choose $f_{X} \in X$ with $f_{X} \notin U$. Then $\left\{f_{X}^{+}\right\}_{X \in X^{\prime}} \in \mathcal{S}$, but $F f_{X}>1$ for all $X \in X^{\prime}$. This is impossible since we have $F f_{X}^{+} \rightarrow 0$ and $1<F f_{X}=F f_{X}^{+}$ $-F f_{X}^{-} \leqq F f_{X}^{+}$. Thus $U \in X$ and $F$ is continuous; i.e. $F$ belongs to the range of $P$.

Conversely, if $F$ belongs to the range of $P, F$ is continuous; hence $F f_{X}^{+} \rightarrow 0$ for each $\left\{f_{X}^{+}\right\}_{X \in X^{\prime}} \in S$ since the sequences in $S$ converge to 0 . This completes the proof.

\section{BIBLIOGRAPHY}

1. H. Bauer, Eine Rieszsche Bandzerlegung im Raum der Bewertungen eines Verbandes, Sitzgber. Math.-Nat. Kl. Bayer. Akad. Wiss. München vol. 1953 (1954) pp. 89-117.

2. - Über die Beziehungen einer abstrackten Theorie des Riemann-Integrals zur Theorie Radonscher Masse, Math. Z. vol. 65 (1956) pp. 448-482.

3. G. Birkhoff, Lattice theory, rev. ed., Amer. Math. Soc. Colloquium Publications, vol. 25, 1948.

4. N. Bourbaki, Espaces vectoriels topologiques, Hermann, Paris, 1953-1955.

5. P. Daniell, A general form of the integral, Ann. of Math. vol. 19 (1917-1918) pp. 279-294.

6. H. Gordon, Compatible topologies on Riesz spaces. Preliminary report, Bull. Amer. Math. Soc. vol. 63 (1957) p. 233.

7. H. Gordon and E. R. Lorch, The projection of a linear functional on the manifold of integrals, Canad. J. Math. vol. 9 (1957) pp. 465-474. 

378.

8. E. R. Lorch, On integration theory, Bull. Amer. Math. Soc. vol. 63 (1957) pp. 377-

9. I. Namioka, Partially ordered linear topological spaces, Memoirs Amer. Math. Soc., vol. 24, 1957.

10. F. Riesz, Sur quelque notions fondamentales dans la theorie generale des operations lineaires, Ann. of Math. vol. 41 (1940) pp. 174-206.

11. G. T. Roberts, Topologies in vector lattices, Proc. Cambridge Philos. Soc. vol. 48 (1952) pp. 533-546.

HARVARD Universuty,

Cambridge, Massachusetts

Columbia University,

NEW YORK, NEW YORK 